\title{
Improving polymeric microemulsions with block copolymer polydispersity
}

Article

Published Version

Thompson, R. B. and Matsen, M. W. (2000) Improving polymeric microemulsions with block copolymer polydispersity. Physical Review Letters, 85 (3). pp. 670-673. ISSN 0031-9007 doi: https://doi.org/10.1103/physrevlett.85.670 Available at https://centaur.reading.ac.uk/63618/

It is advisable to refer to the publisher's version if you intend to cite from the work. See Guidance on citing.

Published version at: http://dx.doi.org/10.1103/physrevlett.85.670

To link to this article DOI: http://dx.doi.org/10.1103/physrevlett.85.670

Publisher: American Physical Society

All outputs in CentAUR are protected by Intellectual Property Rights law, including copyright law. Copyright and IPR is retained by the creators or other copyright holders. Terms and conditions for use of this material are defined in the End User Agreement.

\section{www.reading.ac.uk/centaur}

\section{CentAUR}

Central Archive at the University of Reading

Reading's research outputs online 


\title{
Improving Polymeric Microemulsions with Block Copolymer Polydispersity
}

\author{
R. B. Thompson and M.W. Matsen* \\ Polymer Science Centre, University of Reading, Whiteknights, Reading RG6 6AF, United Kingdom
}

(Received 24 January 2000)

\begin{abstract}
Recent experiments have demonstrated that block copolymers are capable of stabilizing immiscible homopolymer blends producing bicontinuous microemulsion. The stability of these polymeric alloys requires the copolymer to form flexible, nonattractive monolayers along the homopolymer interfaces. We predict that copolymer polydispersity can substantially and simultaneously improve the monolayers in both of these respects. Furthermore, polydispersity should provide similar improvements in systems, such as colloidal suspensions and polymer/clay composites, that utilize polymer brushes to suppress attractive interactions.
\end{abstract}

PACS numbers: 83.80.Es, 81.05.Qk, 82.70.Dd, 83.70.Hq

The opportunity to create new useful materials continues to motivate considerable research on polymeric blends. The difficulty facing researchers is that unlike polymers generally phase separate just like water and oil. This problem can be overcome in the small molecule system by introducing surfactant (i.e., soap) molecules with two distinct ends, generally a polar head group and a hydrocarbon tail, that favor water and oil, respectively. The surfactants self-organize at the water/oil interface forming a flexible monolayer of ultralow tension. This permits the formation of a bicontinuous microemulsion, where the water and oil form interweaving microscopic domains each spanning the system and separated by an extensive amount of internal interface. Polymer scientists have taken a similar strategy by creating $A B$ diblock copolymer compatibilizers, where chains of the two immiscible components (denoted $A$ and $B)$ are bonded together to form a surfactantlike molecule. However, this has not been nearly as successful. Only recently have stable polymeric bicontinuous microemulsions [see Fig. 1(a)] been formed [1], and these only involved slightly immiscible homopolymers. There are two serious problems with copolymer monolayers [see Fig. 1(b)]: they tend to be inflexible and they often attract each other. Recent calculations [2] show that these problems can be reduced by adjusting the copolymer polymerization, $N_{c}$, to be $\sim 80 \%$ of the homopolymer polymerization, $N_{h}$. Such optimization would produce a significant although limited improvement. Here, we demonstrate that far more substantial improvements can be achieved by simply introducing polydispersity into the diblock copolymers. The polydispersity simultaneously improves flexibility and suppresses attractive interactions. Furthermore, this reduced attraction could be utilized to improve numerous other systems such as polymerically stabilized colloidal suspensions [3] and polymer/clay composites [4].

Although bicontinuous microemulsions are new to the polymer community, water/oil microemulsions have been studied for decades. The theories for these mixtures have been based primarily on membrane models [5], in which the energy of the surfactant monolayer is approximated by [6]

$$
F_{\mathrm{int}}=\int d \mathcal{A}\left[\sigma+\frac{1}{2} \kappa\left(c_{1}+c_{2}-2 c_{0}\right)^{2}+\bar{\kappa} c_{1} c_{2}\right]
$$

where $c_{1}$ and $c_{2}$ are the principal curvatures, which vary continuously over the area of the monolayer. The constant elastic coefficients, $\sigma, c_{0}, \kappa$, and $\bar{\kappa}$, are denoted as the interfacial tension, spontaneous curvature, bending modulus, and saddle-splay modulus, respectively, and, in principle, they can be calculated from microscopic models. There are well-accepted conditions which these coefficients must satisfy for the formation of bicontinuous microemulsions. First, an ultralow interfacial tension (i.e., $\sigma \approx 0$ ) is required to allow for the large amounts of interface [7]. Second, a near-zero spontaneous curvature (i.e., $c_{0} \approx 0$ ) is needed, or otherwise the compatibilizer will form micelles rather than a connected surface spanning the entire system $[7,8]$. The former results in a micellar microemulsion, where the composition tends to be highly unbalanced, as opposed to the more desirable bicontinuous microemulsion, where both homopolymers exist in comparable amounts. Finally, bicontinuous microemulsions are stabilized by thermal fluctuations, and therefore their monolayers must be highly flexible (i.e., $|\kappa|$, $\left.|\bar{\kappa}| \lesssim k_{B} T\right)[5,7]$.

In principle, achieving a low interfacial tension is a straightforward matter of adding sufficient compatibilizer to the blend. It is best that low tensions be attained with the minimal amount of compatibilizer possible, since it is generally expensive, but we will not be concerned with that here. In the polymeric system, a near-zero spontaneous curvature is easily achieved by creating symmetric blends, where the $A$ and $B$ homopolymers each contain $N_{h}$ segments and where each block of the copolymer contains $\frac{1}{2} N_{c}$ segments. (Note, we use a standard convention, where the $A$ and $B$ segments are defined based on a common volume, $\rho_{0}^{-1}$.) The stiffness (i.e., $\kappa$ and $\bar{\kappa}$ ) of a saturated (i.e., $\sigma=0$ ) diblock monolayer scales approximately as $\chi^{5 / 6} N_{c}^{4 / 3}$ [9], where $\chi$ is the standard FloryHuggins interaction parameter between $A$ and $B$ segments. 

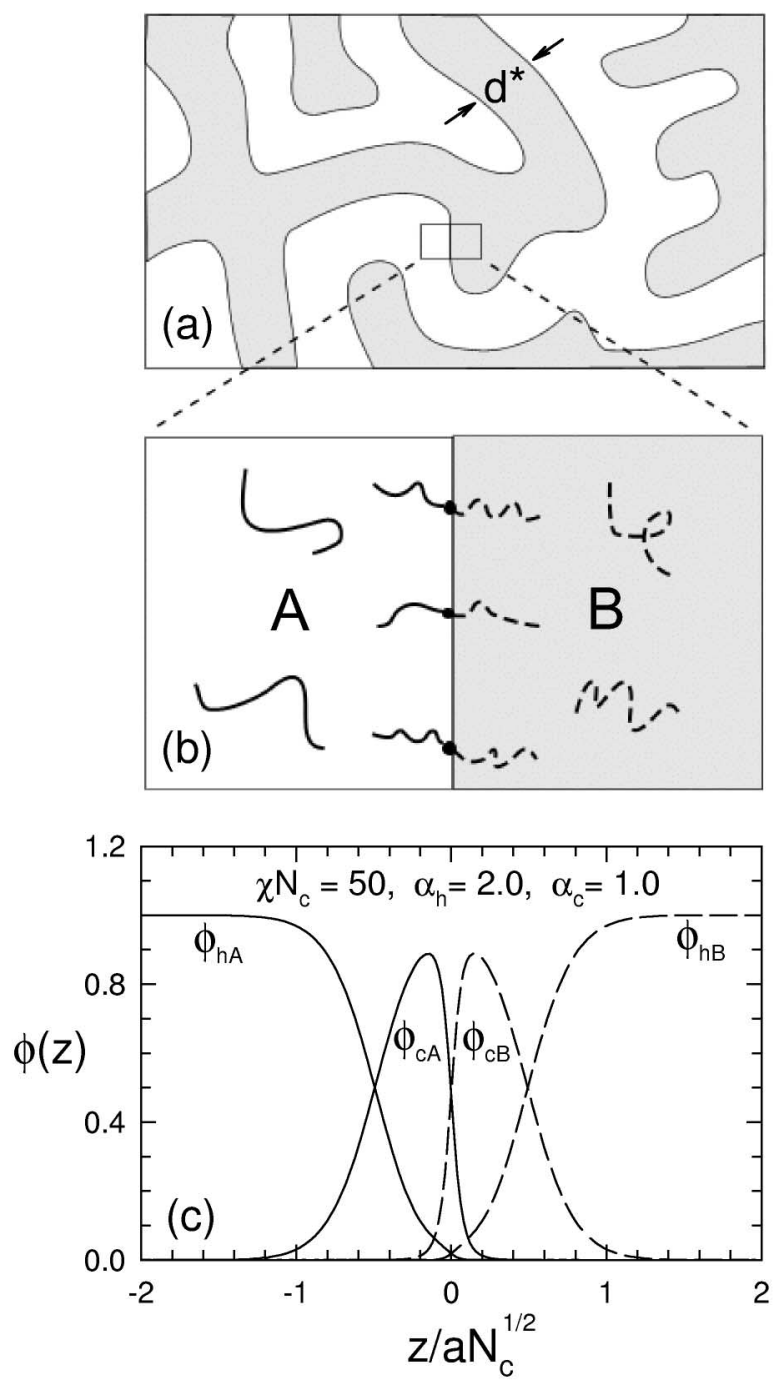

FIG. 1. (a) Schematic diagram of bicontinuous microemulsion, where the immiscible components (denoted $A$ and $B$ ) form interweaving microdomains with an average equilibrium size of $d^{*}$. (b) $A B$ diblock copolymer monolayer separating the $A$ - and $B$-rich homopolymer regions. (c) SCFT calculation showing the two homopolymer concentrations $\phi_{h \nu}(z)$ and the profiles of the two copolymer blocks $\phi_{c \nu}(z)(\nu=A$ or $B)$ for an interface at $z=0$. The interface is defined as the point where $\phi_{h A}(z)+\phi_{c A}(z)=\phi_{h B}(z)+\phi_{c B}(z)$.

Because the flexibility is nearly independent of $N_{h}$ and because $\chi$ is fixed by the temperature and the chemistry of the polymers, the only way to produce an adequately flexible monolayer is to make $N_{c}$ sufficiently small.

For polymeric microemulsions, however, a problem arises, when $N_{c}$ becomes too small relative to $N_{h}$, due to attractive interactions between the monolayers [10]. The interfacial energy $F_{\text {int }}$ of two flat monolayers of area $\mathcal{A}$ separated by a distance $d$ can be expressed as

$$
F_{\text {int }}=\mathcal{A}[2 \sigma+V(d)],
$$

where $V(d)$ is an effective interaction energy per unit area. From that, the force per unit area between the monolayers is $-V^{\prime}(d)$, where positive values imply a repulsion.
If, instead, there exists a sizable attraction, the copolymer monolayers will stick together, forcing out the immiscible homopolymers, thus causing the blend to macrophase separate. Our previous calculations [2] indicate that, to avoid this, $N_{c}$ must remain slightly larger than $N_{h}$. Since the block copolymer also has to be small enough to produce a flexible monolayer, this condition severely limits the molecular weight of the homopolymers. In fact, previous experiments [1] have only produced microemulsions at conditions slightly beyond the critical point (i.e., $\chi N_{h} \gtrsim 2$ ) of the two homopolymers. Had these experiments increased their homopolymer molecular weights to $\alpha_{h} \equiv N_{h} / N_{c} \approx 0.8$, it may have been possible to stabilize blends with $\chi N_{h} \sim 10$. Nevertheless, this Letter proposes a method of producing microemulsions at far higher incompatibilities, where $\chi N_{h} \sim 100$. High homopolymer molecular weights are absolutely essential, if the resulting polymeric alloys are to have good mechanical properties.

Such improvements should be possible provided we can suppress the attraction between the monolayers. With this in mind, we turn our attention to the mechanisms responsible for the interaction between the copolymer monolayers $[9,11]$. It is well established that the attraction originates from the configurational entropy loss experienced by homopolymers when they are confined between two monolayers. In order to recover this entropy, the homopolymers tend to migrate towards bulk homopolymer domains. Consequently, the monolayers are forced together, and hence an effective attraction occurs. Fortunately, the homopolymers gain a significant amount of translational entropy by swelling the copolymer brushes. Because such entropy is lost as the monolayers begin to overlap, this entropy produces an effective repulsion. Since the translational entropy is proportional to the number of homopolymer molecules swelling in the copolymer monolayers, the strength of the repulsion tends to vary inversely with the homopolymer size. Our previous calculation [2] predicts that the repulsion will dominate the attraction provided $\alpha_{h} \equiv N_{h} / N_{c} \lesssim 0.8$.

We previously suggested [2] that the attraction would be suppressed, if the monolayer contained a distribution of copolymer molecular weights. The polydispersity of the copolymer blocks would produce a more gradual interface between the monolayer and the homopolymer. This would, in turn, decrease the loss of configurational entropy experienced by the homopolymers [9,11], and thus would reduce the attractive interaction. Furthermore, we suggested that the polydispersity would increase the flexibility of the monolayers. This latter speculation was based on strongsegregation calculations by Milner et al. [12] showing that polydispersity lowers the stretching energy of a polymer brush. We note that the anionic techniques used to synthesize block copolymers is not well adapted to producing large polydispersities, and so a more practical solution may be to simply mix short and long copolymers. 
In this Letter, we test the above hypotheses by employing self-consistent field theory (SCFT) to examine bimodal monolayers formed from short and long symmetric (i.e., $f=0.5$ ) diblock copolymers of polymerizations, $N_{c, s}$ and $N_{c, l}$, respectively. The copolymer polydispersity (or, more accurately, bidispersity) will be specified using the parameter $\alpha_{c} \equiv N_{c, l} / N_{c, s}$, and the average copolymer polymerization will be defined as $N_{c} \equiv N_{c, l}^{\beta} N_{c, s}^{1-\beta}$, where $\beta \equiv \Omega_{c, l} /\left(\Omega_{c, l}+\Omega_{c, s}\right)$ is expressed in terms of the interfacial excesses of long and short diblock copolymers. These excesses are given by

$$
\Omega_{c, \tau}=\frac{1}{a N_{c}^{1 / 2}} \int_{-\infty}^{\infty} d z\left[\phi_{c, \tau}(z)-\phi_{c, \tau}( \pm \infty)\right]
$$

where $\phi_{c, \tau}(z)$ is the usual dimensionless concentration profile of either the long or short (i.e., $\tau=l$ or $s$ ) copolymer molecules for an isolated monolayer at $z=0$. Although the SCFT calculations are rather involved, they are straightforward generalizations of our previous work [2,9] for monodisperse monolayers (i.e., $\alpha_{c}=1.0$ ). Below, we present our results for monolayers at $\chi N_{c}=50, \alpha_{h} \equiv$ $N_{h} / N_{c}=2.0$, and $\beta=0.5$ as a function of bidispersity $\alpha_{c}$. For this highly immiscible system $\left(\chi N_{h}=100\right)$, the bulk copolymer concentrations, $\phi_{c, \tau}( \pm \infty)$, are effectively zero.

Figure 2 demonstrates a substantial improvement in the monolayer flexibility as the bidispersity $\alpha_{c}$ increases. (The quantity $\bar{N}_{c} \equiv \rho_{0}^{2} a^{6} N_{c}$ is the usual invariant polymerization index; a typical value is $\bar{N}_{c} \sim 10^{3}$ [13].) Of the two elastic coefficients, it is most important that $-\bar{\kappa}$ be of order $k_{B} T$ (see Refs. [5,9,14]), which requires $-\bar{\kappa} / k_{B} T \bar{N}_{c}^{1 / 2} \sim$ 0.03 . At $\chi N_{c}=50$, the monolayer would normally be too stiff, but this is no longer the case once the bidispersity reaches $\alpha_{c}=4.0$.

Figure 3(a) shows the interaction energy $V(d)$ between two monolayers as a function of their separation $d$. For the monodisperse case $\left(\alpha_{c}=1.0\right)$, there is a strong attractive well that would bind the monolayers together at

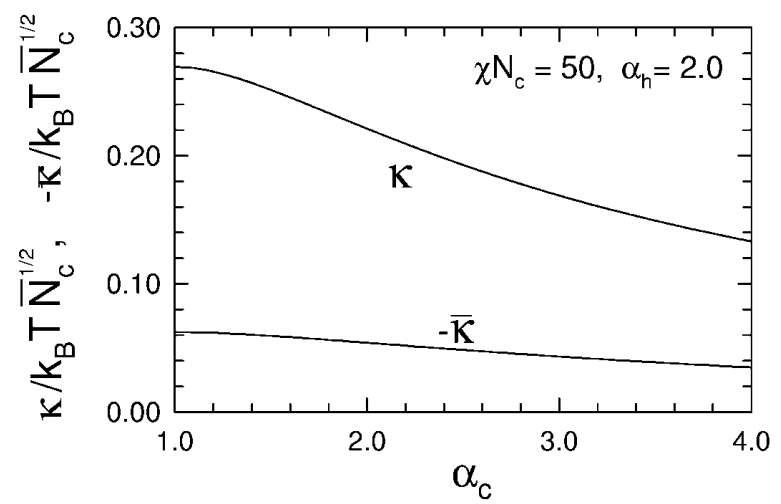

FIG. 2. Bending modulus $\kappa$ and saddle-splay modulus $\bar{\kappa}$ of a diblock copolymer monolayer as a function of bidispersity $\alpha_{c} \equiv N_{c, l} / N_{c, s}$. $d_{\min }=1.10 a N_{c}^{1 / 2}$. However, this attraction is virtually eliminated as the bidispersity reaches $\alpha_{c}=4.0$. Figure 3(b) plots the reduction in the depth of the well with increasing bidispersity. Note that the system can accept a small attraction as long as the monolayer fluctuations are sufficient to keep them out of the well. To assess whether this is the case, we examine the effective repulsion,

$$
V_{\text {fluct }}(d)=\frac{\left(k_{B} T\right)^{2}}{\kappa\left(d-d_{\text {steric }}\right)^{2}},
$$

due to fluctuations calculated by Helfrich [15] for two flexible layers sterically separated by a distance $d_{\text {steric }}$ representing the layer thickness. In order to apply this expression to our bidisperse monolayers at $\alpha_{c}=4.0$, we estimate $d_{\text {steric }} \approx a N^{1 / 2}$, take $\kappa / k_{B} T \bar{N}_{c}^{1 / 2}=0.13$ from Fig. 2, and select a typical value $\bar{N}_{c}=10^{3}$. With these values, $V_{\text {fluct }}(d)$ is sufficient to wipe out the small minimum in $V(d)$ shown in Fig. 3(a).

We have performed analogous calculations over a complete range of experimentally relevant segregations (i.e., $12 \lesssim \chi N_{c} \lesssim 200$ ). Over this range, the attraction can be suppressed up to $\alpha_{h} \approx 2$, whereas for monodisperse monolayers, it is necessary to maintain $\alpha_{h} \lesssim 1$ [2]. Although the monolayer properties improve monotonically with increasing bidispersity, they tend to saturate as $\alpha_{c}$ becomes large. At low segregations, the improvement
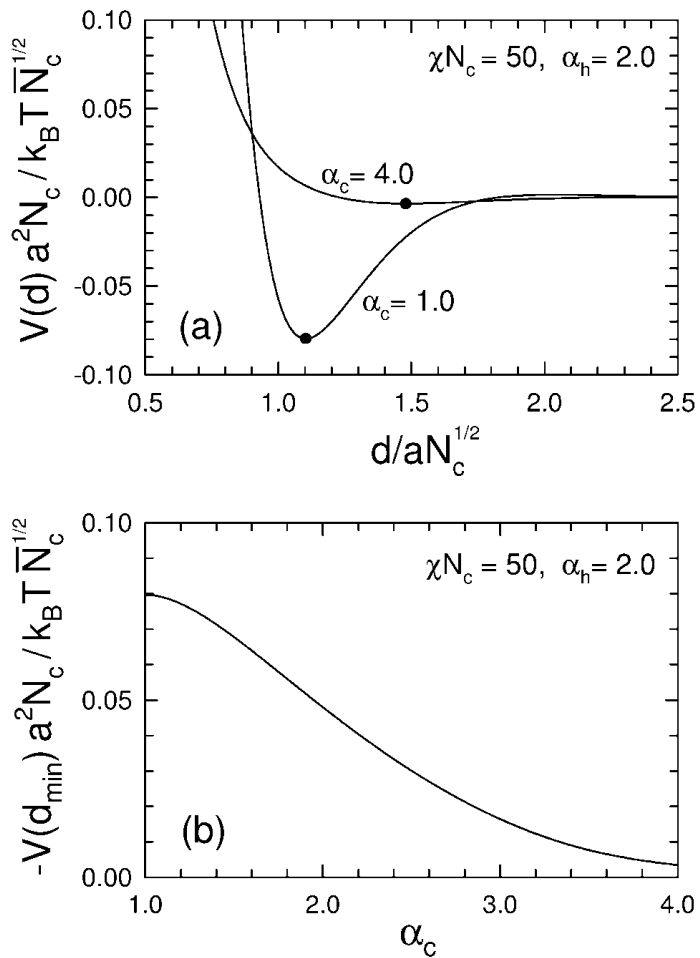

FIG. 3. (a) Interaction energy $V(d)$ as a function of separation $d$ for two monodisperse $\left(\alpha_{c}=1.0\right)$ monolayers and for two bidisperse $\left(\alpha_{c}=4.0\right)$ monolayers. Solid dots denote the position $d_{\min }$ of the attractive wells. (b) Depth of the well $-V\left(d_{\min }\right)$ as a function of the copolymer bidispersity $\alpha_{c}$. 
is ultimately limited by the fact that short copolymers will not segregate to the interface. At stronger segregations considered here, we have to be conscious that the copolymers may begin to segregate within the monolayers if $\alpha_{c}$ exceeds $\sim 5$ (see Ref. [16]). This is an issue that will be considered in a forthcoming publication presenting a more thorough study of this system.

The results presented in this Letter represent the highest homopolymer incompatibility (i.e., $\chi N_{h}=\chi N_{c} \alpha_{h}=$ 100) we have found where the improvements derived from the bidispersity should still be sufficient to form a stable bicontinuous microemulsion. To compare, present experiments [1] have only produced microemulsions with $\chi N_{h} \sim 3$. The material properties of any polymeric alloy created from a microemulsion depends crucially on the ability to compatibilize high-molecular-weight homopolymers, and thus our prediction represents a tremendous improvement. Furthermore, this improvement is absolutely free; it is just as easy to add two molecular weights of diblock copolymer to a blend as it is one. Nevertheless, further advances could be identified by exploring, for example, other molecular-weight distributions or other block copolymer architectures. Besides the obvious commercial incentive for such studies, research of this nature will be important to our basic understanding of microemulsions and other related phases.

The reduced attraction between copolymer monolayers predicted by our calculation offers potential benefits for far more systems than simply polymeric microemulsions. For example, dispersions of colloidal particles are often stabilized against flocculation due to Van der Waals attractions by coating them with polymeric brushes [3]. This is effective when the matrix is a solvent, but this approach can fail when the matrix contains polymer. The presence of high-molecular-weight polymer causes an attraction between the brushes in the same way it does for our copolymer monolayers. Again, this attraction could be suppressed by coating the colloidal particles with polydisperse brushes. Another class of systems that could benefit are the huge variety of composites formed from polymers and small hard (i.e., ceramic or metallic) particles or fibers [4]. The configurational entropy loss experienced by the polymers due to the internal interfaces tends to make the two materials incompatible, but this problem can be overcome by coating the hard particles with polymer brushes [17]. Once again, we suggest that it would be better if the brushes were polydisperse. To test such ideas, we are now applying SCFT to examine the interaction between bidisperse brushes, and our preliminary results confirm our expectations.

This work was supported by the EPSRC (GR/M18034).

*Author to whom correspondence should be addressed.

[1] F. S. Bates et al., Phys. Rev. Lett. 79, 849 (1997); H. S. Joen, J. H. Lee, N. P. Balsara, and M. C. Newstein, Macromolecules 31, 3340 (1998); M. A. Hillmyer et al., J. Phys. Chem. B 103, 4814 (1999); T. L. Morkved et al., Faraday Discuss. 112, 335 (1999); D. Schwahn, K. Mortensen, H. Frielinghaus, and K. Almdal, Phys. Rev. Lett. 82, 5056 (1999); D. Schwahn et al., J. Chem. Phys. 112, 5454 (2000).

[2] R. B. Thompson and M. W. Matsen, J. Chem. Phys. 112, 6863 (2000).

[3] D. H. Napper, Polymeric Stabilization of Colloidal Dispersions (Academic Press, London, 1983).

[4] P.C. LeBaron, Z. Wang, and T. J. Pinnavaia, Appl. Clay Sci. 15, 11 (1999).

[5] D.C. Morse, Curr. Opin. Colloid Interface Sci. 2, 365 (1997).

[6] W. Helfrich, Z Naturforsch. 28C, 693 (1973).

[7] P. G. de Gennes and C. Taupin, J. Phys. Chem. 86, 2294 (1982).

[8] R. Strey, Colloid. Polym. Sci. 272, 1005 (1994).

[9] M. W. Matsen, J. Chem. Phys. 110, 4658 (1999).

[10] A. M. Mayes, T. P. Russell, S. K. Satija, and C. F. Majkrzak, Macromolecules 25, 6523 (1992); K.-J. Jeon and R.-J. Roe, Macromolecules 27, 2439 (1994); H. S. Jeon et al., Macromolecules 30, 973 (1997).

[11] K. R. Shull, J. Chem. Phys. 94, 5723 (1991); A. N. Semenov, Macromolecules 26, 2273 (1993).

[12] S. T. Milner, T.A. Witten, and M.E. Cates, Macromolecules 21, 2610 (1988).

[13] F. S. Bates et al., Faraday Discuss. 98, 7 (1994).

[14] D. C. Morse, Phys. Rev. E 50, R2423 (1994).

[15] W. Helfrich, Z Naturforsch. C 33, 305 (1978).

[16] T. Hashimoto, K. Yamasaki, S. Koizumi, and H. Hasegawa, Macromolecules 26, 2895 (1993); M. W. Matsen, J. Chem. Phys. 103, 3268 (1995); C. M. Papadakis, K. Mortensen, and D. Posselt, Eur. Phys. J. B 4, 325 (1998).

[17] A. C. Balazs, C. Singh, and E. Zhulina, Macromolecules 31, 8370 (1998); A. C. Balazs, C. Singh, E. Zhulina, and Y. Lyatskaya, Acc. Chem. Res. 32, 651 (1999). 